Gut and Liver, Vol. 10, No. 1, January 2016, pp. 58-62

\title{
Clinical Risk Factors for Upper Gastrointestinal Bleeding after Percutaneous Coronary Intervention: A Single-Center Study
}

Ji-Myoung Lee*, Seon-Young Park*, Jung-Ho Choi*, Uh-Jin Kim*, Soo-Jung Rew ${ }^{\dagger}$, Jae Yeong Cho ${ }^{\ddagger}$, Youngkeun Ahn ${ }^{\ddagger}$, SungWook Lim*, Chung-Hwan Jun*, Chang-Hwan Park*, Hyun-Soo Kim*, Sung-Kyu Choi*, and Jong-Sun Rew*

${ }^{*}$ Division of Gastroenterology and Hepatology, Department of Internal Medicine, Chonnam National University Medical School, ${ }^{\dagger}$ Department of Internal Medicine, Gwangju Christian Hospital, and ${ }^{\ddagger}$ Division of Cardiology, Department of Internal Medicine, Chonnam National University Medical School, Gwangju, Korea

Background/Aims: Percutaneous coronary intervention $(\mathrm{PCl})$ is often performed therapeutically, and antithrombotic treatment is required for at least 12 months after stent implantation. However, the development of post-PCl upper gastrointestinal bleeding (UGIB) increases morbidity and mortality. We investigated the incidence and risk factors for UGIB in Korean patients within 1 year after PCl. Methods: The medical records of 3,541 patients who had undergone PCl between January 2006 and June 2012 were retrospectively reviewed. We identified 40 cases of UGIB. We analyzed the incidence and clinical risk factors associated with UGIB occurring within 1 year after $\mathrm{PCl}$ by comparing the results for each case to matched controls. The propensity score matching method using age and sex was utilized. Results: UGIB occurred in 40 patients (1.1\%). Two independent risk factors for UGIB were a history of peptic ulcer disease (odds ratio [OR], 12.68; 95\% confidence interval [Cl], 2.70 to 59.66; $\mathrm{p}=0.001)$ and the use of anticoagulants $(\mathrm{OR}, 7.76 ; 95 \% \mathrm{Cl}$, 2.10 to 28.66; $p=0.002$ ). Conclusions: UGIB after $\mathrm{PCl}$ occurred at a rate of $1.1 \%$ in the study population. Clinicians must remain vigilant for the possibility of $\mathrm{UGIB}$ after $\mathrm{PCl}$ and should consider performing timely endoscopy in patients who have undergone $\mathrm{PCl}$ and are suspected of having an UGIB. (Gut Liver 2016;10:58-62)

Key Words: Gastrointestinal hemorrhage; Percutaneous coronary intervention; Esophagogastroduodenoscopy

\section{INTRODUCTION}

Percutaneous coronary intervention (PCI) is performed on a wide range of patients with coronary artery disease. ${ }^{1}$ Patients undergoing coronary artery stent placement require long-term administration of two or more antithrombotic agents. These agents are used as adjunctive therapy to reduce the risk of ischemic complications, such as in-stent restenosis and stent thrombosis. ${ }^{2}$ The American College of Cardiology, the American Heart Association, and the Society for Cardiovascular Angiography and Interventions recommend dual antithrombotic therapy. In most cases, aspirin combined with a thienopyridine adenosine diphosphate receptor antagonist such as clopidogrel or ticlopidine are administered for at least 12 months after stent placement. ${ }^{3}$ Accordingly, the risk of overall bleeding including access site and gastrointestinal bleeding (GIB) remains a significant problem. ${ }^{4-6}$ The reported incidence of GIB in patients undergoing PCI ranges from $0.4 \%$ to $4.3 \%{ }^{1,2,5,7,8}$ Development of postPCI GIB increases morbidity, hospitalization duration, and inhospital mortality. ${ }^{1,2,5,6}$ Such bleeding complications, most of which arise from the upper gastrointestinal tract, can limit the use of antiplatelet drugs. ${ }^{9}$ There is lack of studies investigating upper GIB (UGIB) after PCI in large study population, especially in Asian patients, and little is known about the incidence, etiology, and clinical risk factors in these patients.

Therefore, the aim of this study was to investigate the incidence and clinical risk factors of UGIB within 1 year after PCI in Korean patients.

\section{MATERIALS AND METHODS}

\section{Study design}

We retrospectively analyzed the data of patients with acute myocardial infarction (AMI) who had visited Chonnam National

Correspondence to: Seon-Young Park

Department of Internal Medicine, Chonnam National University Hospital, Chonnam National University Medical School, 42 Jebong-ro, Dong-gu, Gwangju 61469, Korea

Tel: +82-62-220-6296, Fax: +82-62-225-8578, E-mail: drpsy@naver.com

Received on April 4, 2014. Revised on July 28, 2014. Accepted on October 13, 2014. Published online May 13, 2015

pISSN 1976-2283 eISSN 2005-1212 http://dx.doi.org/10.5009/gnl14127

@) This is an Open Access article distributed under the terms of the Creative Commons Attribution Non-Commercial License (http://creativecommons.org/licenses/by-nc/4.0) which permits unrestricted non-commercial use, distribution, and reproduction in any medium, provided the original work is properly cited. 
University Hospital, Gwangju, Korea, from January 2006 to June 2012.

This study was approved by the Institutional Review Board of Chonnam National University Hospital (IRB number: CNUH2014-018). A total of 3,541 patients with AMI underwent PCI. Among these patients, we identified the patients with clinically significant UGIB within 1 year of the index PCI $(n=40)$. Clinically significant UGIB was defined as hematemesis, melena, hematochezia, or anemia with positive stool occult blood with abnormal upper endoscopic findings. Controls were randomly selected in the same cohort $(3,501$ patients with AMI during the same period) using a simple random sample procedure where each control had equal probability being selected; 10 controls were selected without replacement. Propensity scores were calculated using a logistic regression model and the covariates of age and gender. Using these propensity scores, patients with UGIB after PCI were individually matched to patients without UGIB after PCI using the nearest neighbor matching method. This method consists of randomly ordering case and control subjects and selecting a case subject and finding control subjects with the closet propensity score. ${ }^{10}$ Subsequently, five control patients were matched to each UGIB patient.

We reviewed the medical records of all patients to determine their baseline demographic and clinical characteristics, including age; gender; smoking history; presence of systemic disease such as hypertension, diabetes, or chronic kidney disease; history of peptic ulcer disease (PUD); and indications for PCI. We also reviewed medication history with particular attention to the use of aspirin, clopidogrel, cilostazol, anticoagulants (warfarin/ heparin), nonsteroidal anti-inflammatory drugs (NSAIDs), steroids, proton pump inhibitors (PPIs), histamine 2 receptor antagonist, and antacids. We recorded the length of time between PCI and the onset of bleeding, endoscopic findings, and the management of UGIB.

The primary outcome was to assess the risk factors of UGIB within 1 year after the index PCI. As secondary outcomes, we evaluated the incidence, etiology, success rate of endoscopic hemostasis, rate of recurrent bleeding, and mortality of UGIB.

\section{Statistical analysis}

Continuous variables were presented as means \pm standard deviation; comparisons were conducted by Student t-test or Mann-Whitney U test when group distributions were skewed. Categorical variables were presented as percentages and relative frequencies; comparisons were conducted using chi-square statistics or Fisher exact test as appropriate. The effects of clinical variables on UGIB were estimated by calculating odds ratios (ORs) and 95\% confidence intervals (CIs) using logistic regression analyses. Risk factors that were determined to be significant by univariate analysis were then subjected to multivariate logistic regression analyses. Cumulative UGIB rates were evaluated using the Kaplan-Meier method and compared using the log-rank test. All statistical analyses were performed using IBM SPSS Statistics 18 (IBM Corp., Armonk, NY, USA), and a pvalue of $<0.05$ was considered statistically significant.

\section{RESULTS}

\section{Incidence of UGIB after PCI}

A total of 3,541 patients had undergone PCI between January 2006 and June 2012, among which 40 patients (1.1\%) developed UGIB within 1 year after index PCI. There were no patients with recurrent bleeding within 1 year after index PCI. Fig. 1 illustrates the cumulative incidence of UGIB over 1 year of follow-up. The Kaplan-Meier estimated rate of UGIB at 4 weeks was $0.31 \%$ (95\% CI, 0.11 to 0.51). The estimated rates at 8, 24, and 52 weeks of follow-up were $0.48 \%$ (95\% CI, 0.28 to 0.68), $0.70 \%$ (95\% CI, 0.50 to 0.90 ), and $1.12 \%$ (95\% CI, 0.87 to 1.27), respectively. The monthly incidence of UGIB was $0.08 \%$ per month.

\section{Risk factors for UGIB after PCI}

There were significant differences in advanced age (OR, 1.05; 95\% CI, 1.02 to $1.09 ; \mathrm{p}=0.005$ ), male gender (OR, 4.89; 95\% CI, 1.71 to $14.04 ; \mathrm{p}=0.003$ ), history of PUD (OR, 9.19; 95\% CI, 2.57 to $32.93 ; \mathrm{p}=0.001$ ), the use of anticoagulant therapy (OR, 6.69; 95\% CI, 2.08 to 21.52; $\mathrm{p}=0.001$ ), and NSAID therapy (OR, 32.08; $95 \%$ CI, 1.61 to $641.15 ; \mathrm{p}=0.023$ ) between the 40 patients and the 400 randomized controls.

Table 1 shows the characteristics of 40 cases and 200 controls that were selected using propensity score. There were significant differences in the history of PUD and the use of anticoagulants, NSAIDs, and PPI. Multivariate logistic regression analysis revealed the history of PUD (OR, 12.68; 95\% CI, 2.70 to 59.66; $\mathrm{p}=0.001$ ) and the use of anticoagulants (OR, 7.76; 95\% CI, 2.10 to 28.66; $p=0.002$ ) as independent risk factors for UGIB (Table 2).

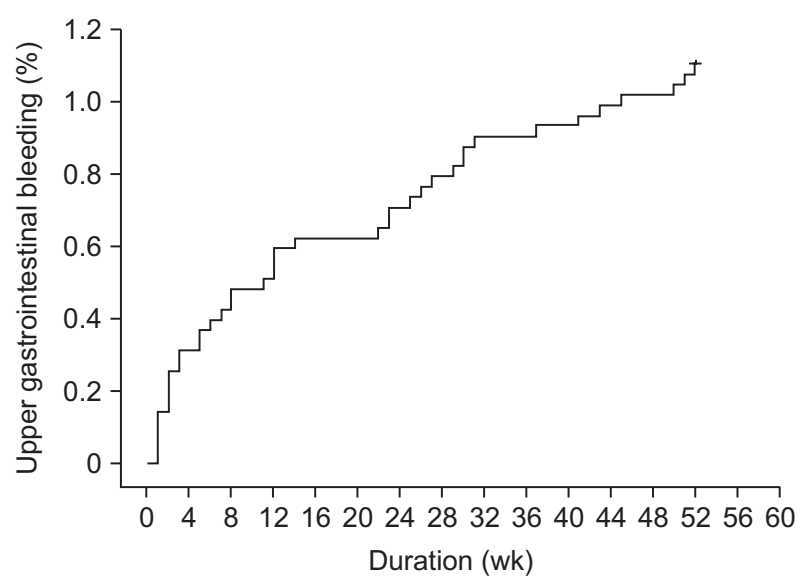

Fig. 1. Cumulative incidence of upper gastrointestinal bleeding over 1 year of follow-up analyzed using the Kaplan-Meier method. 


\section{Mortality}

There was no difference in all-cause mortality rate between cases and controls at 30-day mortality (1/40 [2.5\%] vs $12 / 400$ [3.0\%], p=0.447), 1 year (6/40 [15.0\%] vs 29/400 [7.8\%], $\mathrm{p}=0.126)$. In cases, the cause of death at 1 year could be established in four out of six patients who had UGIB. Three patients died from cardiac causes and one died from advanced gastric cancer. Medical records were not available for two patients who died after hospital discharge.

Table 1. Clinical Characteristics of Cases and Age- and Sex-Matched Controls

\begin{tabular}{lccc}
\hline \multicolumn{1}{c}{ Characteristic } & $\begin{array}{c}\text { Cases } \\
(\mathrm{n}=40)\end{array}$ & $\begin{array}{c}\text { Controls } \\
(\mathrm{n}=200)\end{array}$ & $\mathrm{p}$-value \\
\hline Age, yr & $69.2 \pm 10.7$ & $69.0 \pm 10.2$ & 0.898 \\
Male sex & $35(87.5)$ & $175(87.5)$ & 1.000 \\
Smoker & $21(52.5)$ & $130(65.0)$ & 0.135 \\
Presence of systemic disease & & & \\
$\quad$ Hypertension & $26(65.0)$ & $107(53.5)$ & 0.182 \\
$\quad$ Diabetes & $12(30.0)$ & $57(28.5)$ & 0.848 \\
$\quad$ Chronic kidney disease & $8(20.0)$ & $20(10.0)$ & 0.072 \\
History of peptic ulcer disease & $6(15.0)$ & $3(1.5)$ & $<0.001$ \\
Indications for PCI & & & 0.450 \\
$\quad$ STEMI & $20(50.0)$ & $113(56.5)$ & \\
$\quad$ NSTEMI & $20(50.0)$ & $87(43.5)$ & \\
Drug use & & & \\
Aspirin & $39(97.5)$ & $198(99.0)$ & 0.436 \\
Clopidogrel & $38(95.0)$ & $192(96.0)$ & 0.773 \\
Cilostazol & $20(50.0)$ & $96(48.0)$ & 0.817 \\
Anticoagulants (warfarin/heparin) & $7(17.5)$ & $5(2.5)$ & $<0.001$ \\
NSAIDs & $2(5.0)$ & $1(0.5)$ & 0.019 \\
Proton pump inhibitor & 0 & $21(10.5)$ & 0.032 \\
Other gastrointestinal protector & $37(92.5)$ & $165(82.5)$ & 0.114 \\
\hline Da & & & \\
\hline
\end{tabular}

Data are presented as mean \pm SD or number $(\%)$.

PCI, percutaneous coronary intervention; STEMI, ST-segment elevation myocardial infarction; NSTEMI, non-ST-segment elevation myocardial infarction; NSAIDs, nonsteroidal anti-inflammatory drugs.

\section{Etiology of UGIB after PCI}

Endoscopic examinations were performed on all 40 patients with UGIB. Endoscopic findings showed a wide range of etiologies for UGIB (Table 3). Fifteen patients (37.5\%) had endoscopic lesions indicating high risk of recurrent bleeding (peptic ulcers with active bleeding, exposed vessels, or adherent blood clots). Endoscopic hemostasis was done in 19 patients by epinephrine injection $(n=15)$, argon plasma coagulation therapy for ablation $(\mathrm{n}=7)$, and clipping $(\mathrm{n}=10)$. Endoscopic hemostasis was 100\% successful in preventing recurrent bleeding.

\section{DISCUSSION}

This study is the first large-scale study of UGIB after PCI in Asian patients. The incidence of UGIB within 1 year after PCI was $1.1 \%$. Univariate analysis showed that advanced age, male gender, previous history of PUD, anticoagulant therapy, and NSAID therapy were risk factors for the development of UGIB after PCI. Multivariate analysis showed that previous history of PUD and the use of anticoagulant were independent risk factors. Although endoscopy revealed a wide range of etiologies for UGIB, the most common cause was peptic ulcer (85\%). The success rate of endoscopic hemostasis was 100\%, and there were no deaths associated with UGIB.

Previous studies have shown that the rate of any type of GI bleeding after PCI was $0.4 \%$ to $3.3 \% .^{1,2,5,7-9,11,12}$ In the pres-

Table 3. Etiology of Upper Gastrointestinal Bleeding $(n=40)$

\begin{tabular}{lc}
\multicolumn{1}{c}{ Etiology } & No. (\%) \\
\hline GU & $27(67.5)$ \\
Gastric erosion & $2(5.0)$ \\
Gastric polyp & $1(2.5)$ \\
Gastric malignancy & $1(2.5)$ \\
Hemorrhagic gastritis & $1(2.5)$ \\
DU & $7(17.5)$ \\
Mallory-Weiss tear & $1(2.5)$ \\
\hline
\end{tabular}

GU, gastric ulcer; DU, duodenal ulcer.

Table 2. Independent Risk Factors for Upper Gastrointestinal Bleeding after Percutaneous Coronary Intervention in Patients and Age- and SexMatched Controls

\begin{tabular}{|c|c|c|c|c|c|c|}
\hline \multirow{2}{*}{ Factor } & \multicolumn{3}{|c|}{ Univariate } & \multicolumn{3}{|c|}{ Multivariate } \\
\hline & $\mathrm{aOR}$ & $95 \% \mathrm{CI}$ & p-value & $\mathrm{aOR}$ & $95 \% \mathrm{CI}$ & p-value \\
\hline Hypertension & 1.61 & $0.80-3.27$ & 0.182 & 1.26 & $0.57-2.78$ & 0.575 \\
\hline CKD & 2.25 & $0.91-5.55$ & 0.072 & 1.09 & $0.36-3.37$ & 0.875 \\
\hline PUD & 11.59 & $2.77-48.57$ & $<0.001$ & 12.68 & $2.70-59.66$ & 0.001 \\
\hline Use of anticoagulants & 8.27 & $2.48-27.62$ & $<0.001$ & 7.76 & $2.10-28.66$ & 0.002 \\
\hline NSAIDs & 10.47 & $0.93-118.42$ & 0.019 & 8.87 & $0.65-121.44$ & 0.102 \\
\hline
\end{tabular}

aOR, adjusted odds ratio; CI, confidence interval; CKD, chronic kidney disease; PUD, peptic ulcer disease; NSAIDs, nonsteroidal anti-inflammatory drugs. 
ent study, the incidence rate of UGIB was similar to that noted in these previous studies. The present study also shows that 13 cases (0.4\%) of UGIB occurred within 4 weeks of PCI. Chin et $a{ }^{9}{ }^{9}$ found that the incidence of UGIB was $1.2 \%$ within 30 days. Several reports have indicated that the incidence of major bleeding within 1 month of PCI was between $0.6 \%$ and 2.3\%, indicating that the first month after PCI seems to be the period of high risk for UGIB. ${ }^{9,13}$ However, in our study, the incidence of UGIB was $0.08 \%$ per month and the probability of UGIB increased over time. Physicians need to be vigilant for UGIB after PCI, especially in patients with associated risk factors, and endoscopy should be performed in patients when there is a possibility of UGIB. This present study showed that there was no difference in all-cause mortality rate between cases and controls at 30-day (2.5\% vs 3.0\%, p=0.447) and 1-year (15.0\% vs 7.8\%, $\mathrm{p}=0.126)$. Chin's study showed the difference in mortality rate between cases and controls at $30(11.9 \%$ vs $0.5 \%, p=0.0041)$ and 180 days $(13.4 \%$ vs $0.5 \%, p=0.001)$. Chin's study showed the difference of reason for PCI between cases and controls. There were significantly more cases than controls undergoing primary PCI for AMI (34.3\% vs 8.5\%) and conversely fewer cases undergoing PCI for stable angina as compared with controls (6.0\% vs 26.3\%). However, in this present study, cases and controls were all diagnosed AMI. Therefore, the difference of mortality rate in Chin's study might be caused by underlying cardiovascular disease status.

We found that male gender, advanced age, previous history of PUD, use of anticoagulants, and use of NSAIDs were independent risk factors for UGIB after PCI. These risk factors for UGIB were similar to those suggested by previous studies. ${ }^{1,2}$ In the Acute Catheterization and Urgent Intervention Triage Strategy trial, ${ }^{11}$ advanced age, baseline anemia, longer duration of study drug administration before angiogram, smoking, STsegment deviation $>1 \mathrm{~mm}$, and diabetes were identified as independent predictors of GIB. In a study by Chin et al., ${ }^{9}$ risk factors for UGIB were prior PCI, cardiac arrest, inotropic drug support, thienopyridine use before PCI, and advanced age. In the present study, we used the propensity score matching method to objectively compare the clinical risk factors of UGIB in well-matched groups. Propensity score methods are being increasingly used to reduce the impact of selection bias when retrospective data is used to assess clinical factors. ${ }^{10}$ Accordingly, in the present study, cases and controls were individually matched with respect to age and gender. Analysis of the data comparing UGIB patients' age and gender with that of matched controls showed that previous history of PUD and use of anticoagulants remained independent risk factors for UGIB. Therefore, it is imperative that the clinician maintain a high level of vigilance in patients with a history of PUD or the prior use of anticoagulants or NSAIDs. Moreover, a thorough drug history is very important and the discontinuation or modification of high-risk medications should be considered if clinically possible.
In the current study, the most common etiology of UGIB after index PCI was PUD. PPI is indicated in patients at high risk including those with the history of PUD, multiple NSAID use, anticoagulant use, and advanced age. ${ }^{14,15}$ During period of present study, PPIs for prophylaxis of UGIB were not routinely prescribed. Furthermore, no patients with UGIB after PCI had received PPIs beforehand. The cardiologist may be afraid of using PPI in the initial period of this study because of inhibition of antiplatelet action. Therefore there is a limitation to analyze the effect of PPIs for prevention of UGIB in patients with PCI. ${ }^{15}$

We found that among the 40 UGIB patients who underwent endoscopy after PCI, no patient developed complications from the endoscopic procedure. We also found that 37.5\% patients had endoscopic lesions at high risk of recurrent bleeding. These endoscopic findings heightened the clinicians' awareness and vigilance for possible recurrent bleeding. Furthermore, the success rate of endoscopic hemostasis was 100\%. This demonstrates the diagnostic and therapeutic benefits of the relatively low-risk endoscopy procedure.

Our study has several limitations. First, our study was retrospective study with the potential for selection bias. All data were collected by chart review. Outcomes were subjectively assessed based on documentation in the clinical record. Secondly, we did not routinely test for Helicobacter pylori infection. Earlier reports have shown that the prevalence of $H$. pylori infection is high in Korea, especially in the older population. ${ }^{16} \mathrm{H}$. pylori infection is thought to increase the risk of GI bleeding in patients receiving antiplatelet therapy. ${ }^{15,16}$ A prospective and multicenter study with a larger cohort of patients is needed in the future so that the risk factors and protective factors can be defined more precisely. Further, an observational or prospective study can be performed using the database of the ongoing registries such as Korea Acute Myocardial Infarction Registry, the most recent and largest AMI database in Korea launched in November 2005, which is the first nationwide, population-based, multicenter data collection registry in Korea designed to measure outcomes of patients with AMI.

In conclusion, UGIB after PCI was $1.1 \%$ in the study population. It is necessary for clinicians to remain vigilant for the possibility of UGIB after PCI, especially in the elderly, male patients, those with a history of PUD, and those on anticoagulants or NSAIDs. Clinicians should always keep in mind that timely endoscopy might be needed in patients with high risk for UGIB. A prospective and multicenter study with a larger cohort of patients is needed in the future so that the risk factors and protective factors can be defined more precisely.

\section{CONFLICTS OF INTEREST}

No potential conflict of interest relevant to this article was reported. 


\section{REFERENCES}

1. Tanigawa T, Watanabe T, Nadatani Y, et al. Gastrointestinal bleeding after percutaneous coronary intervention. Digestion 2011;83: 153-160.

2. Nadatani Y, Watanabe T, Tanigawa T, et al. Incidence and risk factors of gastrointestinal bleeding in patients on low-dose aspirin therapy after percutaneous coronary intervention in Japan. Scand J Gastroenterol 2013;48:320-325.

3. Kushner FG, Hand M, Smith SC Jr, et al. 2009 focused updates: ACC/AHA guidelines for the management of patients with STelevation myocardial infarction (updating the 2004 guideline and 2007 focused update) and ACC/AHA/SCAI guidelines on percutaneous coronary intervention (updating the 2005 guideline and 2007 focused update) a report of the American College of Cardiology Foundation/American Heart Association Task Force on Practice Guidelines. J Am Coll Cardiol 2009;54:2205-2241.

4. Eikelboom JW, Mehta SR, Anand SS, Xie C, Fox KA, Yusuf S. Adverse impact of bleeding on prognosis in patients with acute coronary syndromes. Circulation 2006;114:774-782.

5. Nikolsky E, Mehran R, Stone GW. Gastrointestinal bleeding in percutaneous coronary intervention and acute coronary syndromes. Am J Cardiol 2009;104(5 Suppl):22C-29C.

6. Rao SV, O'Grady K, Pieper KS, et al. Impact of bleeding severity on clinical outcomes among patients with acute coronary syndromes. Am J Cardiol 2005;96:1200-1206.

7. Ng FH, Wong SY, Lam KF, et al. Gastrointestinal bleeding in patients receiving a combination of aspirin, clopidogrel, and enoxaparin in acute coronary syndrome. Am J Gastroenterol 2008; 103:865-871.

8. Toyokawa T, Inaba T, Ishikawa S, Nakatsu M, Ando M, Tomoda J. Investigation of upper gastrointestinal bleeding after implantation of drug-eluting stents: prospective cohort study. Scand J Gastroenterol 2010;45:1097-1100.
9. Chin MW, Yong G, Bulsara MK, Rankin J, Forbes GM. Predictive and protective factors associated with upper gastrointestinal bleeding after percutaneous coronary intervention: a case-control study. Am J Gastroenterol 2007;102:2411-2416.

10. D’Agostino RB Jr. Propensity score methods for bias reduction in the comparison of a treatment to a non-randomized control group. Stat Med 1998;17:2265-2281.

11. Nikolsky E, Stone GW, Kirtane AJ, et al. Gastrointestinal bleeding in patients with acute coronary syndromes: incidence, predictors, and clinical implications. Analysis from the ACUITY (Acute Catheterization and Urgent Intervention Triage Strategy) trial. J Am Coll Cardiol 2009;54:1293-1302.

12. Almadi MA, Barkun A, Brophy J. Antiplatelet and anticoagulant therapy in patients with gastrointestinal bleeding: an 86-year-old woman with peptic ulcer disease. JAMA 2011;306:2367-2374.

13. Abbas AE, Brodie B, Dixon S, et al. Incidence and prognostic impact of gastrointestinal bleeding after percutaneous coronary intervention for acute myocardial infarction. Am J Cardiol 2005;96: 173-176.

14. Lai KC, Lam SK, Chu KM, et al. Lansoprazole for the prevention of recurrences of ulcer complications from long-term low-dose aspirin use. N Engl J Med 2002;346:2033-2038.

15. Abraham NS, Hlatky MA, Antman EM, et al. ACCF/ACG/AHA 2010 expert consensus document on the concomitant use of proton pump inhibitors and thienopyridines: a focused update of the ACCF/ACG/AHA 2008 expert consensus document on reducing the gastrointestinal risks of antiplatelet therapy and NSAID use. A report of the American College of Cardiology Foundation Task Force on Expert Consensus Documents. J Am Coll Cardiol 2010; 56:2051-2066.

16. Lim SH, Kwon JW, Kim N, et al. Prevalence and risk factors of Helicobacter pylori infection in Korea: nationwide multicenter study over 13 years. BMC Gastroenterol 2013;13:104. 